\title{
When Physical and Social Distances Produce An Analogical Perceptual Bias in the Ebbinghaus Illusion
}

\author{
Kévin Moinier \\ Juliette Gasquet \\ Vincent Murday \\ Lionel Brunel \\ Laboratoire Epsylon (EA 4556) - Université Paul-Valéry - Montpellier 3 - France
}

\begin{abstract}
Grounded theory argues that perceptual and memory processes share common sensorimotor properties, and that they influence each other during perceptual processing of the environment's features. When these principles are applied to social cognition, it was shown that to live, or represent, a situation related to a social distance concept (e.g., ostracism) leads to a similar bias on the perceptual judgements of the space's properties, illustrating that distance-physical cues are intrinsically linked to social concepts. In two experiments using an Ebbinghaus illusion based-paradigm, we investigated the symmetrical incidence produced by a perceptual physical (Experiment 1) and conceptual social distance (Experiment 2) on the perceptual judgements of size. The present findings have shown an analogical pattern of results, regardless of whether the perceived distance between the central and inducer disks was physically or conceptually manipulated. Experiment 1 indicated that when the physical distance between these latter disks was important, the size-contrast perceptual bias was weaker. Experiment 2 has shown a similar weakness of the Ebbinghaus illusion when the social distance was present between the central and inducer disks. A plausible explanation for both sets of findings is that insofar as social distance concepts are physically based, it appears that a perceptual dimension of physical distance can be reactivated by the presence of a conceptual social distance between stimuli. As a consequence, it is not surprizing that a analogical size-contrast perceptual bias emerges when a perceptual physical distance and conceptual social distance are inserted in Ebbinghaus illusion figures.
\end{abstract}

Keywords: Grounded social cognition; physical and social distances; Ebbinghaus illusion paradigm; size-contrast perceptual bias.

\section{Introduction}

Grounded theory argues that perceptual and conceptual processes share common sensorimotor properties and they influence each other, in such manner that memory can bias perceptual processing and perception can bias memory processing (Barsalou, 2008; Goldstone, de Leeuw, \& Landy, 2015; van Dantzig, Pecher, Zeelenberg, \& Barsalou, 2008). This reciprocal influence has notably been shown in the processing of perceptual components (Brunel et al., 2009, 2010; Riou, Rey, Vallet, Cuny, \& Versace, 2015), as well as perceptual judgments of an object's size (Rey et al., 2014, 2015). Moreover, a grounded perspective assumes that situations in which these cognitive activities are involved are not independent of the emergent sensorimotor state, insofar as a given situation, lived or represented (i.e., simulated), is an integral part of these cognitive activities (Barsalou, 2008; Niedenthal, Barsalou, Winkielman, Krauth-Gruber, \& Ric, 2005; Semin \& Smith, 2013). Accordingly, when these principles are applied to the social cognition, it is not surprising to observe that to experiment or represent a social situation, such as ostracism (Zhong \& Leonardelli, 2008) or feeling of social power (Yap, Mason, \& Ames, 2013), leads to a similar perceptual bias of physical features of the environment or others.

The idea that concepts related to social situations can bias our perception of the physical space, as well as judgements of an object's properties, is known in social cognition literature (see e.g., Morgado, Muller, Gentaz, \& Palluel-Germain, 2011; Lee \& Schnall, 2014; Schnall, Harber, Stefanucci, \& Proffitt, 2008). More particularly, it appears that physical-distance cues 
are intrinsically linked to social distance concepts (Knowles, Green, \& Weidel, 2014; Williams \& Bargh, 2008). For instance, an ostracized individual is both socially and physically kept away from the ostracizing group; this why an ostracized individual tend to perceive the rejecting people as more distant than accepting ones (Knowles et al., 2014). In the same vein, it was shown that to live or represent an experiment of social power leads to perceive others as smaller (Yap et al., 2013), which is not surprising insofar as the social concept of power is embodied within a vertical spatial dimension (Robinson, Zabelina, Ode, \& Moeller, 2008; Schubert, 2005). In this respect, it is reasonable to think that it could exist a symmetrical perceptual bias between a perceptual physical distance and conceptual social distance. The present study aims to examine this analogical influence on the judgements of an object's size by using an Ebbinghaus illusion basedparadigm, which has been shown as an useful tool to investigate the reciprocity of perceptual and memory processing (see Rey et al., 2014, 2015).

\section{// Insert Figure 1 about here //}

The Ebbinghaus illusion paradigm is known to highlight a perceptual bias when two identical central disks are simultaneously displayed, which are surrounded by either smaller or larger inducers (see Figure 1). Although central disks have the same size, participants usually report that they are not. The Ebbinghaus illusion is typically explained as resulting of a size-contrast perceptual bias induced by the inducers' size, in such a way that an underestimation of the central disk's size is observed when inducers are large, and an overestimation when they are smaller (Coren \& Enns, 1993; Massaro \& Anderson, 1971). Recently, it was shown that this perceptual bias is not only perceptually-based and that it may be reactivated in memory (Rey, Riou, \& Versace, 2014). Rey et al. (2014) have adapted the Ebbinghaus illusion paradigm by creating a color-size association during a learning phase. For instance, large inducers disks were associated to the red colour and the small ones to the blue colour. In the test phase, the inducers disks were displayed in a physically identical size, but in different colours such as previously presented (i.e., red or blue). Their results indicated that the learned colorsize associations have biased the perceptual judgements of size, in such way that the inducers' size has been influenced by the reactivated memory size (for similar results, see Rey, Vallet, Riou, Lesourd, \& Versace, 2015).

In the same vein, other studies have stressed that the magnitude of the Ebbinghaus illusion is also modulated by the conceptual similarity between the Ebbinghaus figure's components (e.g., Coren \& Enns, 1993). For instance, Ishii \& Kitayama (2011) have used the out-group homogeneity effect (i.e., the tendency to perceive out-group members as more similar than in-group ones, see Boldry, Gaertner, \& Quinn, 2007 for a review) in order to show that a phenomenon related to categorical similarity can affect the size-contrast perceptual bias. To this end, the authors have inserted pictures of American adults' faces in the central and inducers disks, and manipulated the group membership by associating a specific background colour to disks (red background was associated to the out-group members, and the blue background to the ingroup ones). Similarly to classical results, the perceived size of the central-target face was overestimated when the surrounding inducers-faces was small. More interestingly, Ishii and Kitayama (2011) shown that the size-contrast perceptual bias was greater when the central-target face was enclosed by inducers-faces of out-group members (i.e., red background).

Along these lines, it appears that perceptual and conceptual dimensions of an object modulate the perceptual judgment of its size. However, could these latter dimensions lead to a symmetrical size-contrast perceptual bias? Distance-physical cues appear to be deeply linked to social concepts (Knowles et al., 2014; Robinson et al., 2008; Schubert, 2005; Williams \& Bargh, 2008); if so, the distance between components of the Ebbinghaus illusion figure, whether it is perceptually or conceptually based, should leading to an analogical perceptual bias. To test this assumption, we used an Ebbinghaus illusion based-paradigm, in which the size-contrast perceptual bias was induced either by a perceptual physical distance (Experiment 1) or conceptual social distance (Experiment 2) between the central and inducers disks. We expected that a symmetrical influence should occur on the perceptual judgments of size. More precisely, the magnitude of the Ebbinghaus illusion should be weaker when the physical distance between central and inducers disks is important, in a similar way to when the social distance between these latter disks is present. 


\section{Experiment 1: Physical distance between central and inducer disks.}

\section{Method}

\section{Participants}

Sixteen undergraduate students from Paul Valery Montpellier 3 University, France, volunteered to take part in the experiment. All participants have freely given consent for their participation and reported to have a normal or corrected-tonormal vision.

\section{Stimuli \& Apparatus}

Stimuli were based on the traditional configuration of the Ebbinghaus illusion paradigm. Each stimulus consisted to the simultaneous presentation of two black central disks symmetrically positioned on each side of the screen, one enclosed by six large black equally spaced inducers and another by six small ones. Two different central (i.e., $1.91^{\circ}$ and $2.865^{\circ}$ for the small and large diameter respectively) and inducers sizes (i.e., $2.387^{\circ}$ and $3.342^{\circ}$ for the small and large diameter respectively), as well as three different physical distances separating these latter disks were investigated (i.e., $3.342^{\circ}$, $4.297^{\circ}$, and $4.966^{\circ}$ for the short, medium, and grand distance respectively, which were measured from the centre of the target-central disk to the centre of the inducers). These manipulations lead to a total of twelve configurations ( 2 central disk sizes $\times 2$ inducer disk sizes $\times 3$ physical distances).

Participants were positioned to $60 \mathrm{~cm}$ in front of the 17-inch ASUS X75A laptop (ASUSTeK Computer Inc.) used to conduct both experiments. Opensesame 3.0.7 (Mathôt, Schreij, \& Theeuwes, 2012) was used to set up the experiments and for data collection.

\section{Procedure}

After completing and signing a written consent form, the participants were informed that they going to perform a perceptual discrimination task. Participants were asked to indicate whether the size of target-central disks was identical or different, by pressing an appropriate key on an AZERTY keyboard. The key " $m$ " and "q" were attributed to the "identical" and "different" response, respectively. This configuration of keys was counterbalanced between participants.

Each trial began with the appearance of a fixation-cross that was presented for $1000 \mathrm{~ms}$ at the centre of the screen. Then, stimulus was displayed for $400 \mathrm{~ms}$, immediately followed by a blank screen that was presented until participant's response. The inter-trial interval was set at $1500 \mathrm{~ms}$ (see Figure 2a). The twelve Ebbinghaus illusion configurations were repeated twice in a block of 24 trials, insofar as the size of the inducers was counterbalanced on each side of the screen. The 24trial block was repeated six times in a random order, for a total of 144 trials. The duration of an experimental session was approximately 15 minutes.

// Insert Figure 2 a,b about here //

\section{Statistical analyses}

The mean percentages of hits (e.g., participant indicated "different" when target-central disks were presented with different sizes) and false alarms (FA, e.g., participant responded "different" when target-central disks were displayed with an identical size), as well as a signal detection quality index ( $d^{\prime}$ ) were calculated per participant and per physical distance condition (see Table 1). The $d^{\prime}$ indicator corresponds to the signal's strength relative to the noise, the more is higher and the better was the participant's ability to discriminate the central disks' size. The $d^{\prime}$ 'was calculated as $d^{\prime}$ index $=z$-value proportion of hits Z-value proportion of false alarms (see Macmillan \& Creelman, 2005).

Table 1. Mean percentages of hits and FA, and the $d^{\prime}$ 'indicator for each physical distance condition.

\begin{tabular}{llll}
\hline & \multicolumn{2}{l}{ Physical distance } & Grand \\
\cline { 2 - 4 } & Short & Medium & $0.84(0.19)$ \\
\hline Hit & $0.86(0.16)$ & $0.88(0.16)$ & $0.15(0.12)$ \\
FA & $0.21(0.20)$ & $0.15(0.19)$ & 2.864 \\
$d^{\prime}$ & 2.158 & 2.968 & \\
\hline
\end{tabular}


Note. Standard deviations are in parentheses.

For both experiments, statistical analyses were performed with $\mathrm{R}$ software (version 3.2.2; R Core Team, 2015). The residuals' normality of our dependent variables has been verified by means of the shapiro.test function (stats $\mathrm{R}$ package), excepted the d's distribution for the "grand" physical distance $(W=.97$ and $p=.82)$, all were $W<.89$ and $p<.08$. As a consequence, we opted for non-parametric alternative analyses by using the friedman.test ${ }^{1}$ and wilcox.test functions (stats R package).

\section{Results and Discussion}

To test the incidence of the physical distance on the magnitude of the Ebbinghaus illusion, a Friedman test was conducted to compare differences in the participants' $d$ ' scores across the three conditions of physical distances (i.e., short, medium, and large). As expected, the analysis provided evidence for a significant effect of the physical distance on the participants' discrimination quality, $X^{2}(2)=7.23, p=.027$. Post hoc Wilcoxon signed-rank tests (paired method) showed that it was more difficult to discriminate the size of the central-target disks when the distance separating them from inducers disks was short compared to medium, $V=15.5, p=.012$, and the large one, $V=29, p=.046$. In contrast, no significant difference was found between medium and large distances, $V=50.5, p=.753$.

Also, two separate Friedman tests were performed on the participants' hits and FA scores according to the three physical distance conditions. No significant effect was found for hits, $X^{2}(2)=3.71, p=.156$, and FA, $X^{2}(2)=3.11, p=.211$. However, it is important to stress that post hoc Wilcoxon signed-rank tests (paired method) showed that the participants committed more of FA when the physical distance between central and inducer disks was short compared to medium, $V=69.5, p=$ .018. No further effect was highlighted, all $V>18.5$, and $p>.09$.

Similarly to prior Roberts et al.'s study (2005), Experiment 1 shown that the size-contrast perceptual bias is influenced by the physical distance between central and inducer disks. The participants' discrimination ability was weaker when the physical distance between these latter disks was short rather medium or large. In order to assess whether a conceptual social distance produces an analogical perceptual bias than this observed for the physical distance, Experiment 2 employed the same paradigm as Experiment 1 with two exceptions. First, pictures were inserted in the Ebbinghaus illusion figures. Second, the physical distance between central and inducer disks was fixed.

\section{Experiment 2: Conceptual social distance in the Ebbinghaus illusion figure.}

\section{Method}

\section{Participants}

Sixteen undergraduate students from Paul Valery Montpellier 3 University, France, volunteered to take part in the experiment. All participants have freely given consent for their participation and reported to have a normal or corrected-tonormal vision.

\section{Stimuli \& Apparatus}

The central and inducers disks were identical to those in Experiment 1 with the exception that we varied the conceptual social distance between these latter disks. To this end, we used two pairs of pictures representing either a "patient and doctor" or "jester and king" association. The size of the central (i.e., $1.91^{\circ}$ and $2.865^{\circ}$ for the small and large diameter respectively) and inducer disks (i.e., $2.387^{\circ}$ and $3.342^{\circ}$ for the small and large diameter respectively) was manipulated, as well as the presence of a social distance between them (i.e., present and absent), for a total of sixteen configurations ( 2 central disk sizes $\times 2$ inducer disk sizes $\times 2$ social distance situations $\times 2$ pairs of pictures). The physical distance between central and inducers disks was constant (i.e., 4.297", "medium" distance in Experiment 1).

\section{Procedure}

The procedure was identical to Experiment 1. The sixteen Ebbinghaus illusion configurations were repeated twice in a block of 32 trials, which 32 -trial block was repeated four times in a random order, for a total of 128 trials. The "patient-doctor" and

\footnotetext{
1 The Friedman test is employed as the non-parametric alternative of the one-way repeated-measures ANOVA design.
} 
"jester-king" picture associations were presented separately; no doctor or patient was surrounded by the jesters or kings pictures, and reciprocally (see Figure $2 b$ ). The duration of an experimental session was approximately 15 minutes.

\section{Results \& Discussion}

As in Experiment 1, the mean percentages of hits and FA, as well as the $d^{\prime}$ indicator were calculated per participant and per social distance condition (see Table 2). The residual's normality of our dependent variables was verified with the shapiro.test $\mathrm{R}$ function, all $\mathrm{W}<.94$, and $p<.06$. In this respect, Wilcoxon signed-rank tests (paired method) were used as non-parametric alternative to the $t$-test.

Table 2. Mean percentages of hits and FA, and the $d^{\prime}$ ' indicator for each social distance condition.

\begin{tabular}{lll}
\hline & \multicolumn{2}{l}{ Social distance } \\
\cline { 2 - 3 } & Present & Absent \\
\hline Hit & $0.85(0.16)$ & $0.83(0.20)$ \\
FA & $0.21(0.28)$ & $0.23(0.28)$ \\
$d^{\prime}$ & 2.514 & 2.091 \\
\hline
\end{tabular}

Note. Standard deviations are in parentheses.

As expected, the Wilcoxon signed-rank test performed on the participants' $d$ ' scores have shown that participants were better to discriminate the size of target-central disks when the social distance between central and inducer disks was present, $\mathrm{V}=86, p=.038$. No evidence of this influence was found for the participants' hits, $\mathrm{V}=53, p=.282$, and $\mathrm{FA}, \mathrm{V}=$ $24.5, p=.149$.

\section{General discussion}

The present study investigated the symmetrical incidence produced by a perceptual physical distance and conceptual social distance on the perceptual judgements of size. According to the grounded theory, memory and perceptual processes influence each other during the perceptual processing of the environment's properties. Previous works applied these principles to the social cognition and have shown that experimenting, or representing, a social situation (e.g., ostracism, social power) lead to a similar perceptual bias of the space and other's physical features. In this respect, we adapted the Ebbinghaus illusion paradigm in two experiments, in order to show that a symmetrical size-contrast perceptual bias can be obtained when a physical distance (Experiment 1) or social distance (Experiment 2) separates the central and inducer disks.

Our findings have shown that regardless of whether the distance between these latter disks was physically or conceptually manipulated, an analogical pattern of results have been observed on the participants' discrimination quality. In Experiment 1 , the more the physical distance separating the central and inducer disks was important, the better and accurate were participants to discriminate the size of the target-central disks (for similar results, see Robert et al., 2005). Similarly, Experiment 2 showed that the size-contrast perceptual bias was weaker when a social distance was present between these latter disks. On this point, our findings diverge from those of Ishii and Kitayama (2011). The present results of Experiment 2 seem to indicate that when a social distance is present between the illustrations of the central and inducer disks, participants are less sensitive to perceptual bias induced by the Ebbinghaus illusion. While in their study, Ishii and Kitayama have shown that the amplitude of the Ebbinghaus illusion tends to increase when the target-central face is surrounded by inducer-faces of out-group members. The interpersonal similarity (e.g., social closeness, group membership) is known as a social distance dimension (Liviatan, Trope, \& Liberman, 2008). Accordingly, participants should be more accurate to discriminate the size of the central disk when it is enclosed by outgroup members rather than ingroup ones, insofar as the perceived interpersonal similarity is minimal between these latter disks. Our results are in line with this logic; furthermore, prior studies have also highlighted such findings (Coren \& Enns, 1993).

The present results are consistent with a grounded perspective arguing that perceptual and memory processes share common sensorimotor properties (for a review, see Barsalou, 2008). Following the grounded principles, physical-distance cues are intrinsically related to social distance concepts (Williams \& Bargh, 2008). For instance, a social distance is typically identified by a vertical spatial dimension (e.g., social power; Schubert, 2005), and well often metaphorically expressed such as "the decisions are taken by people in above us", or "they are above average". In this sense, insofar as the social distance 
concepts are physically based, it is reasonable to think that a perceptual dimension of physical distance can be reactivated by the presence of a conceptual social distance between stimuli. Therefore, it is not surprizing that a symmetrical sizecontrast perceptual bias emerges when a perceptual physical distance and conceptual social distance is inserted are Ebbinghaus illusion figures.

\section{Authors' notes}

Correspondence regarding this article should be addressed to Kevin Moinier - Laboratoire Epsylon (EA 4556) - Université Paul-Valéry Site St-Charles - Route de Mende - 34199 Montpellier - FRANCE

We thank Benjamin Glaviaux for his help in the data collection.

\section{References}

[1] Barsalou, L. W. (2008). Grounded cognition. Annual Review of Psychology, 59, 617-645. doi:10.1146/annurev.psych.59.103006.093639

[2] Boldry, J. G., Gaertner, L., \& Quinn, J. (2007). Measuring the measures: A meta-analytic investigation of the measures of outgroup homogeneity. Group Processes \& Intergroup Relations, 10(2), 157-178. doi:10.1177/1368430207075153

[3] Brunel, L., Labeye, E., Lesourd, M., \& Versace, R. (2009). The sensory nature of episodic memory: Sensory priming effects due to memory trace activation. Journal of Experimental Psychology: Learning, Memory, and Cognition, 35(4), 1081-1088. doi:10.1037/a0015537

[4] Brunel, L., Lesourd, M., Labeye, E., \& Versace, R. (2010). The sensory nature of knowledge: Sensory priming effects in semantic categorization. The Quarterly Journal of Experimental Psychology, 63(5), 955-964. doi:10.1080/17470210903134369

[5] Coren, S., \& Enns, J. T. (1993). Size contrast as a function of conceptual similarity between test and inducers. Perception \& Psychophysics, 54(5), 579-588. doi:10.3758/BF03211782

[6] Goldstone, R. L., de Leeuw, J. R., \& Landy, D. H. (2015). Fitting perception in and to cognition. Cognition, 135, 24-29. doi:10.1016/j.cognition.2014.11.027

[7] Knowles, M. L., Green, A., \& Weidel, A. (2014). Social rejection biases estimates of interpersonal distance. Social Psychological and Personality Science, 5(2), 158-167. doi:10.1177/1948550613491972

[8] Ishii, K., \& Kitayama, S. (2011). Outgroup homogeneity effect in perception: An exploration with Ebbinghaus illusion. Asian Journal of Social Psychology, 14(2), 159-163. doi:10.1111/j.1467-839X.2010.01339.x

[9] Lee, E. H., \& Schnall, S. (2014). The influence of social power on weight perception. Journal of Experimental Psychology: General, 143(4), 1719-1725. doi:10.1037/a0035699

[10] Liviatan, I., Trope, Y., \& Liberman, N. (2008). Interpersonal similarity as a social distance dimension: Implications for perception of others' actions. Journal of Experimental Social Psychology, 44(5), 1256-1269. doi:10.1016/j.jesp.2008.04.007

[11] Macmillan, N. A., \& Creelman, C. D. (2005). Detection theory: A user's guide., 2nd ed. Mahwah, NJ, US: Lawrence Erlbaum Associates Publishers.

[12] Massaro, D. W., \& Anderson, N. H. (1971). Judgmental model of the Ebbinghaus illusion. Journal Of Experimental Psychology, 89(1), 147-151. doi:10.1037/h0031158

[13] Mathôt, S., Schreij, D., \& Theeuwes, J. (2012). OpenSesame: An open-source, graphical experiment builder for the social sciences. Behavior Research Methods, 44(2), 314-324. doi:10.3758/s13428-011-0168-7

[14] Morgado, N., Muller, D., Gentaz, E., \& Palluel-Germain, R. (2011). Close to me? The influence of affective closeness on space perception. Perception, 40(7), 877-879. doi:10.1068/p6830

[15] Niedenthal, P. M., Barsalou, L. W., Winkielman, P., Krauth-Gruber, S., \& Ric, F. (2005). Embodiment in Attitudes, Social Perception, and Emotion. Personality and Social Psychology Review, 9(3), 184-211. doi:10.1207/s15327957pspr0903_1

[16] R Core Team (2015). R: A language and environment for statistical computing. R Foundation for Statistical Computing, Vienna, Austria. https://www.R-project.org/.

[17] Rey, A. E., Riou, B., \& Versace, R. (2014). Demonstration of an Ebbinghaus illusion at a memory level: Manipulation of the memory size and not the perceptual size. Experimental Psychology, 61(5), 378-384. doi:10.1027/1618-3169/a000258 
[18] Rey, A. E., Vallet, G. T., Riou, B., Lesourd, M., \& Versace, R. (2015). Memory plays tricks on me: Perceptual bias induced by memory reactivated size in Ebbinghaus illusion. Acta Psychologica, 161, 104-109. doi:10.1016/j.actpsy.2015.08.011

[19] Riou, B., Rey, A. E., Vallet, G. T., Cuny, C., \& Versace, R. (2015). Perceptual processing affects the reactivation of a sensory dimension during a categorization task. The Quarterly Journal of Experimental Psychology, 68(6), 1223-1230. doi:10.1080/17470218.2014.978876

[20] Roberts, B., Harris, M. G., \& Yates, T. A. (2005). The roles of inducer size and distance in the Ebbinghaus illusion (Titchener circles). Perception, 34(7), 847-856. doi:10.1068/p5273

[21] Robinson, M. D., Zabelina, D. L., Ode, S., \& Moeller, S. K. (2008). The vertical nature of dominancesubmission: Individual differences in vertical attention. Journal of Research in Personality, 42(4), 933-948. doi:10.1016/j.jp.2007.12.002

[22] Schnall, S., Harber, K. D., Stefanucci, J. K., \& Proffitt, D. R. (2008). Social support and the perception of geographical slant. Journal of Experimental Social Psychology, 44(5), 1246-1255. doi:10.1016/j.jesp.2008.04.011

[23] Schubert, T. W. (2005). Your Highness: Vertical Positions as Perceptual Symbols of Power. Journal of Personality and Social Psychology, 89(1), 1-21. doi:10.1037/0022-3514.89.1.1

[24] Semin, G. R., \& Smith, E. R. (2013). Socially situated cognition in perspective. Social Cognition, 31(2), 125146. doi:10.1521/soco.2013.31.2.125

[25] van Dantzig, S., Pecher, D., Zeelenberg, R., \& Barsalou, L. W. (2008). Perceptual processing affects conceptual processing. Cognitive Science, 32(3), 579-590. doi:10.1080/03640210802035365

[26] Williams, L. E., \& Bargh, J. A. (2008). Keeping one's distance: The influence of spatial distance cues on affect and evaluation. Psychological Science, 19(3), 302-308. doi:10.1111/j.1467-9280.2008.02084.x

[27] Yap, A. J., Mason, M. F., \& Ames, D. R. (2013). The powerful size others down: The link between power and estimates of others' size. Journal of Experimental Social Psychology, 49(3), 591-594. doi:10.1016/j.jesp.2012.10.003

[28] Zhong, C., \& Leonardelli, G. J. (2008). Cold and lonely: Does social exclusion literally feel cold?. Psychological Science, 19(9), 838-842. doi:10.1111/j.1467-9280.2008.02165.x

\section{Figure Captions}

Figure 1. Illustration of the classical Ebbinghaus illusion paradigm (target-central disks have the same size).

Figure $2(a, b)$. (a) Example of an "identical" trial with the grand (left side) and small (right side) perceptual physical distances. (b) Example of an "identical" trial when the social distance is present between the king-jester pictures association.

Figure 1

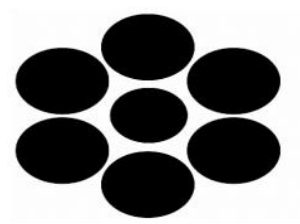

Figure $2 a, b$

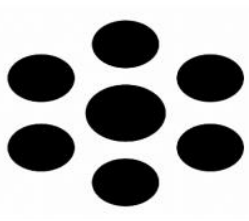

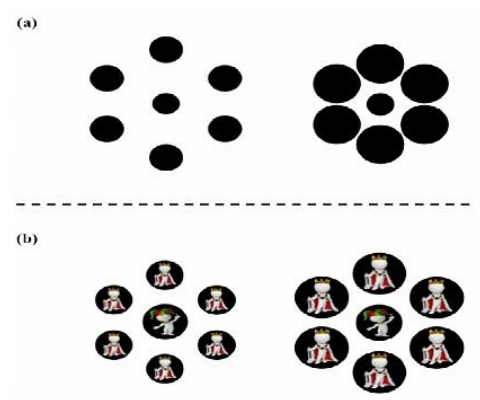

